\title{
ASSOCIATION BETWEEN THE PULSATILITY INDEX OF THE AORTIC ISTHMUS AND THE CARDIAC FUNCTION OF THE LEFT VENTRICLE IN FETUSES BELOW THE PERCENTILE THREE FOR THE GESTATIONAL AGE.
}

Authors: Malka M. Wadnipar, Juan Carlos Otero Pinto

Submitted:

2. February 2019

Published:

Volume:

Issue:

5. February 2019

6

Affiliation:

Languages:

Keywords:

Categories:

2

Universidad Industrial de Santander

Spanish, Castilian

Intrauterine Growth Restriction, Aortic isthmus, Tei index, myocardial performance index, Prenatal Doppler ultrasound Medicine

DOI: $\quad$ 10.17160/josha.6.2.532

\section{Abstract:}

Background: The evaluation of the fetal circulation, mainly of the umbilical artery (AU), middle cerebral artery (MCA) and ductus venosus (DV), have been the pillars in the diagnosis and monitoring of the main alterations in fetuses with restriction of the intrauterine growth (IUGR). Given the need to have a parameter with the predictive power of adverse outcomes that allows us to define the opportune moment of birth of a fetus with IUGR, the pulsatility index of the aortic isthmus appears as the point of connection between the two fetal circulatory systems arranged in parallel between the left and right ventricle. Objective: To establish the degree of correlation between the pulsatility index of the fetal aortic isthmus and the function of the middle left ventricle by the TEI index in fetuses with estimated fetal weight below the 3rd percentile (PFE $<$ p3).

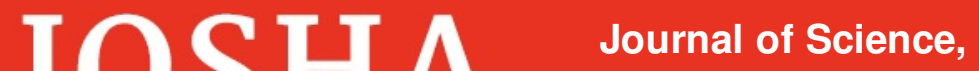 Humanities and Arts}


ASSOCIATION BETWEEN THE PULSATILITY INDEX OF THE AORTIC ISTHMUS AND THE CARDIAC FUNCTION OF THE LEFT VENTRICLE IN FETOS BELOW THE PERCENTILE THREE FOR THE GESTATIONAL AGE.

\title{
ASOCIACIÓN ENTRE EL ÍNDICE DE PULSATILIDAD DEL ISTMO AÓRTICO Y LA FUNCIÓN CARDÍACA DEL VENTRÍCULO IZQUIERDO EN FETOS POR DEBAJO DEL PERCENTIL TRES PARA LA EDAD GESTACIONAL.
}

\author{
Malka Irina Wadnipar Gutiérrez* \\ Juan Carlos Otero Pinto**
}

* Medica Residente de Ginecología y Obstetricia de tercer año, Departamento de Ginecología y Obstetricia, Universidad Industrial de Santander (UIS), Bucaramanga, Colombia. Escuela de Medicina. Dirección de correspondencia: Carrera 32 Nº 29-31, Teléfono: (57) (7) 6324929 -6343496, correo electrónico: malka_159@hotmail.com.

** Especialista en Medicina Materno Fetal, Docente de catedra del Departamento de Ginecología y Obstetricia, Universidad Industrial de Santander, Bucaramanga, Colombia. Dirección de correspondencia: Carrera 32 № 29-31, Teléfono: (57) (7) 6324929 - 6343496, correo electrónico: juan_carlos_otero@yahoo.com. 


\section{RESUMEN}

Antecedentes: La valoración de la circulación fetal, principalmente de la arteria umbilical (AU), arteria cerebral media (ACM) y ductus venoso (DV), han sido los pilares en el diagnóstico y seguimiento de las principales alteraciones en los fetos con restricción del crecimiento intrauterino (RCIU). Ante la necesidad de tener un parámetro con poder de predicción de desenlaces adversos que permita definir el momento oportuno de nacimiento de un feto con RCIU, aparece el índice de pulsatilidad del istmo aórtico como el punto de conexión entre los dos sistemas circulatorios fetales dispuestos en paralelo entre el ventrículo izquierdo y el derecho.

Objetivo: Establecer el grado de correlación entre el índice de pulsatilidad del istmo aórtico fetal y la función del ventrículo izquierdo media por el índice de TEI en fetos con peso fetal estimado por debajo del percentil 3 (PFE $<$ p3).

Diseño del estudio: Estudio descriptivo de cohorte en fetos de gestaciones entre la 24-37 semanas con peso fetal estimado menor de percentil 3 (PFE >p3).

Resultados: En la unidad maternofetal del HUS durante el 2018, se realizó doppler fetal a 1123 gestantes y en 305 se les diagnosticó fetos con RCIU severo lo que corresponde al 27,1\% de nuestra población, siendo el 96\% (293) estadio I, el 1\% (5) estadio II, el 3\% (7) estadio III y no se encontró ninguna paciente con doppler estadio IV. Se encontró que existe una correlación lineal positiva entre el índice de pulsatilidad del istmo aórtico fetal y el índice de TEI de 17,07\%. Es decir, a medida que aumenta el istmo aórtico aumenta el ITEI. A su vez, se encontró que a medida que incrementa el estadio Doppler hay un aumento en la correlación entre las dos variables analizadas, siendo en el estadio I de 4,94\%, en el estadio II es de 41,61 \% y en el estadio III $80 \%$. Al analizar cada uno de los estadios se pudo estimar un coeficiente de determinación (R2) de: 0.001 para el estadio I, de 0.13 para el estadio II y de 0.54 para el estadio III. De acuerdo con el valor de coeficiente de determinación $\left(\mathrm{R}^{2}\right)$ la proporción de varianza es compartida por ambas variables, siendo para el estadio I pequeña, 
en el estadio II media/moderada y en el estadio III fuerte, lo que reafirma la correlación de las dos variables.

Conclusión: Pudimos establecer que existe una correlación positiva entre los valores del índice de pulsatilidad del istmo aórtico fetal y de la función del ventrículo izquierdo en fetos con peso fetal estimado por debajo del percentil 3 (PFE < p3). A su vez, se encontró que a medida que incrementa el estadio Doppler hay un aumento en la correlación entre las dos variables analizadas. Se considera necesario continuar con esta línea de investigación para establecer el papel predictivo de desenlace adverso al usar esta correlación.

Palabras clave: restricción del crecimiento intrauterino, Istmo aórtico, índice de Tei, índice de Rendimiento Miocárdico, Doppler fetal.

\section{SUMMARY}

Background: The evaluation of the fetal circulation, mainly of the umbilical artery (AU), middle cerebral artery (MCA) and ductus venosus (DV), have been the pillars in the diagnosis and monitoring of the main alterations in fetuses with restriction of the intrauterine growth (IUGR). Given the need to have a parameter with predictive power of adverse outcomes that allows us to define the opportune moment of birth of a fetus with IUGR, the pulsatility index of the aortic isthmus appears as the point of connection between the two fetal circulatory systems arranged in parallel between the left and right ventricle.

Objective: To establish the degree of correlation between the pulsatility index of the fetal aortic isthmus and the function of the middle left ventricle by the TEI index in fetuses with estimated fetal weight below the 3rd percentile (PFE <p3).

Study design: Descriptive cohort study in fetuses of gestations between 24-37 weeks with estimated fetal weight lower than percentile 3 (PFE> p3). 
Results: In the maternal-fetal unit of the HUS during 2018, fetal doppler was performed on 1123 pregnant women and 305 were diagnosed with fetuses with severe IUGR corresponding to $27.1 \%$ of our population, being $96 \%$ (293) stage I , 1\% (5) stage II, 3\% (7) stage III and no patient with stage IV Doppler was found. It was found that there is a positive linear correlation between the pulsatility index of the fetal aortic isthmus and the TEI index of $17.07 \%$. That is, as the aortic isthmus increases, the ITEI increases. In turn, it was found that as the Doppler stage increases there is an increase in the correlation between the two variables analyzed, being in stage I of $4.94 \%$, in stage II it is $41.61 \%$ and in the Stage III $80 \%$. By analyzing each of the stages it was possible to estimate a coefficient of determination (R2) of: 0.001 for stage I, 0.13 for stage II and 0.54 for stage III. According to the value of coefficient of determination (R2), the proportion of variance is shared by both variables, being for stage I small, stage II medium / moderate and stage III strong, which reaffirms the correlation of two variables.

Conclusions: We could establish that there is a positive correlation between the values of the pulsatility index of the fetal aortic isthmus and the function of the left ventricle in fetuses with estimated fetal weight below the 3rd percentile ( $\mathrm{PFE}<\mathrm{p} 3$ ). In turn, it was found that as the Doppler stage increases there is an increase in the correlation between the two variables analyzed. It is considered necessary to continue with this line of research to establish the predictive role of adverse outcome when using this correlation.

Key words: Intrauterine Growth Restriction, Aortic isthmus, Tei index, myocardial performance index, Prenatal Doppler ultrasound 


\section{INTRODUCCIÓN}

Los avances en el conocimiento de la fisiopatología de los embarazos con restricción del crecimiento intrauterino (RCIU) y el progreso en el conocimiento la fisiopatología de la de la hipoxia fetal, han inducido al profesional en Medicina Materno-Fetal a buscar medidas útiles y objetivas para predecir el momento óptimo en el que el feto debe nacer, para de esta manera disminuir el riesgo de muerte fetal o de desarrollar secuelas permanentes al nacimiento. Uno de estos avances es el estudio de la circulación fetal, ya que a través de ella podemos identificar variables que predicen el comportamiento que hay entre la circulación materna y fetal (1). La evaluación de la función placentaria por Doppler de las arterias uterina y umbilical es el estándar clínico utilizado para valorar la evolución y pronóstico de los fetos con RCIU, asociado al estudio de los vasos como la arteria cerebral media y el ductus venoso (2).

En el estudio de la hemodinamia fetal, es importante la fase de redistribución de flujo a nivel cerebral, que comprende un fenómeno compensatorio que protege al cerebro de daño neurológico; sin embargo, es de destacar que cuando esta redistribución termina comprometiendo otros vasos como el ductus venoso, nos está expresando que la acidosis fetal está instaurada y, por ende, el daño neurológico en el recién nacido se va a hacer evidente (3). El istmo aórtico, también forma parte de este estudio ya que por su localización anatómica, pues es el punto de conexión entre los dos sistemas circulatorios fetales dispuestos en paralelo entre el ventrículo izquierdo y el derecho (4).

A pesar de todos los avances, no disponemos de un criterio eficaz que pueda establecer cuando el feto se encuentra sobre el límite de la descompensación cardiaca, ya que es este quien lidera el mecanismo de adaptación y el que redistribuye el gasto cardiaco, para conservar la función de diferentes órganos.

Él istmo aórtico y la función cardiaca han sido correlacionadas en distintos estudios (2)(5)(6)(7) para evaluar el problema a resolver. Por esto, nuestro objetivo es el análisis comparativo de las alteraciones de los valores de la onda de flujo del istmo aórtico, junto con 
los cambios iniciales en la función cardiaca medida por el índice de TEI, ya que podrían indicar una correlación positiva, en primera instancia con relación directa o asociativa con el deterioro del bienestar fetal, lo que determinaría en consecuencia la indicación ,con estos parámetros, de la extracción fetal antes de la alteración del ductus venoso (4).

\section{MATERIALES Y MÉTODOS}

Entre marzo del 2018 y enero del 2019, en la Unidad Materno-fetal del Hospital Universitario de Santander, se realizó un estudio descriptivo de cohorte en todas las gestantes (incluyendo menores de edad) con fetos entre la 24-37 semanas con peso fetal estimado menor de percentil 3 (PFE >p3). Se incluyeron solo fetos de gestación única. Los criterios de exclusión fueron: cromosomopatías o malformaciones congénitas que alteren la función cardíaca y neonatos con peso al nacer adecuado para la edad gestacional. El protocolo fue aprobado por el Comité de Ética en Investigación Científica UIS (CEINCI-UIS) y el comité de ética del Hospital Universitario de Santander, con el fin de permitir la realización de la investigación y el acceso a la historia clínica de las pacientes que autoricen, ya sea por medio del consentimiento informado o por el asentimiento informado en caso de las menores de edad.

Las gestantes que cumplieron con los criterios de inclusión se les realizó un Doppler Fetal por especialistas en Medicina Materno Fetal, con las embarazadas en decúbito supino, con ecógrafos GE Vóluson E6 y E8, usando un transductor de 3.5 o $5 \mathrm{MHz}$ y el filtro de paso alto se fijó en $100 \mathrm{~Hz}$. Se realizaron técnicas específicas para la realización de cada vaso estudiado de la siguiente manera: Todas las mediciones espectrales de Doppler se realizaron automáticamente a partir de tres o más formas de onda consecutivas, con el ángulo de isonación siempre inferior a $\operatorname{los} 30^{\circ}$, en ausencia de movimientos fetales y, si es necesario, con la respiración materna suspendida voluntariamente. Para la evaluación de la arteria umbilical, se usó asa libre por facilidad técnica. La evaluación de la arteria cerebral media se realizó en un corte axial craneal a nivel del polígono de Willis, observándose su trayecto de unos 2-4cm desde su parte proximal en la salida en la carótida interna hasta su parte distal adyacente al hueso parietal. Se realizó el calculo la relación cerebroplacentaria (RCP) como 
la relación del IP de la arteria cerebral media/ el IP de la arteria umbilical. El ductus venoso se midió en un plano sagital medio del abdomen fetal en su punto de origen a partir de la vena umbilical o en un plano transverso oblicuo del abdomen fetal entre el corazón y la cámara gástrica en el punto más próximo a la salida de la umbilical. Para la evaluación de las arterias uterinas se colocó el transductor en los cuadrantes inferiores derechos e izquierdos de la pared materna abdominal, donde se visualizó la arteria iliaca externa y se identificó la arteria uterina medial a ella. Las ondas de velocidad de flujo se obtuvieron de cada arteria uterina cerca de la arteria iliaca externa antes de la división de la arteria uterina.

El istmo aórtico fue evaluado ubicando el volumen de muestra en la aorta, debajo de la salida de la arteria subclavia izquierda, se puede realizar en dos planos según la posición fetal: un corte transversal a nivel del mediastino superior, paralelo al plano tetracameral, junto a la vena cava superior y la tráquea identificar la confluencia anatómica del istmo aórtico y el ductus arterioso en forma de V; o un corte longitudinal con una vista del tórax fetal sagitalmente con visualización del segmento vascular del arco localizado entre la salida de la arteria subclavia izquierda y la unidad del ductus arterioso.

Finalmente, el índice de TEI se midió al obtenerse una imagen de la sección transversal del tórax fetal en la vista de cuatro cámaras y una proyección apical del corazón; el volumen de la muestra Doppler se colocó en la pared lateral de la aorta ascendente, por debajo de la válvula aortica (AV) y justo encima de la válvula mitral (VM). La traza Doppler mostró un claro eco de las formas de ondas correspondientes a la apertura y el cierre de las dos válvulas al principio y al final de la onda A (la válvula mitral) y flujo aórtico (válvula aórtica). Las imágenes se registraron utilizando un volumen de muestra de $3 \mathrm{~mm}$, un nivel de ganancia de 60, una velocidad de barrido Doppler de 8 y con la forma de onda E / A siempre mostrada como flujo positivo. Los períodos de tiempo se calcularon de la siguiente manera: el tiempo de contracción isovolumétrico (ICT) se estimó a partir del cierre de la válvula mitral, a la apertura de la válvula aórtica , el tiempo de eyección (ET) desde la apertura hasta el cierre de la AV y el tiempo de relajación isovolumétrico (IRT) desde el cierre de la AV para la 
apertura de la MV de acuerdo con la fórmula MPI = (TIC + IRT $) / \mathrm{ET}$.

Inicialmente los datos obtenidos fueron digitados en una base de datos en formato Excel, se realizó una descripción de todas las características de las variables según su naturaleza: proporciones en las variables cualitativas y promedio o mediana (junto con desviación estándar o recorrido intercuartil) dependiendo de la presencia o ausencia de distribución Gaussiana.

Respecto a los datos obtenidos del Doppler fetal durante el seguimiento de la población, se digitaron los valores reportados por los ecógrafos del índice de pulsatilidad de los vasos estudiados y el MPI; para identificar si se encuentran dentro de límites normales según la edad gestacional se asignó el percentil. Todos los parámetros Doppler se convirtieron en Z scores según las referencias publicadas normales y cualquier con valores por encima del percentil 95 (+ 1.645 Z-score) se consideraron anormales; el IP de la cerebral media cuando se encontró por debajo del percentil 5, se consideró indicativos de la redistribución del flujo sanguíneo cerebral.

Posteriormente para establecer el grado de diferencias entre las variables continuas y cualitativas, se compararon utilizando la prueba estadística t-Student y las variables cualitativas con la prueba de chi cuadrado de Pearson o la prueba exacta de Fisher, aceptando como significativo una $\mathrm{p}<0,05$. Finalmente, se estimó el grado de correlación entre los valores dados por el Doppler por análisis de correlación de Pearson y Spearman.

\section{RESULTADOS}

En la unidad maternofetal del HUS durante el 2018, se realizó doppler fetal a 1123 gestantes y en 305 se les diagnosticó fetos con RCIU severo lo que corresponde al 27,1\% de nuestra población, siendo el 96\% (293) estadio I, el 1\% (5) estadio II, el 3\% (7) estadio III y no se encontró ninguna paciente con doppler estadio IV (Tabla 1). 


\section{JOSHA}

De la población elegible 224 cumplían los criterios de inclusión, sin embargo 20 gestantes no aceptaron participar en el estudio. Se captaron 57 pacientes en total, se logró completar el seguimiento en 51 pacientes; 6 se censuraron, 3 pacientes se perdieron y 3 fueron atendidas fuera de la institución (figura 1). En total se obtuvieron 90 mediciones, el 73\% de las gestantes fueron evaluadas en mínimo 2 ocasiones, el $25 \%$ fue valorada con una sola medición y solo el $2 \%$ contó con 5 mediciones.

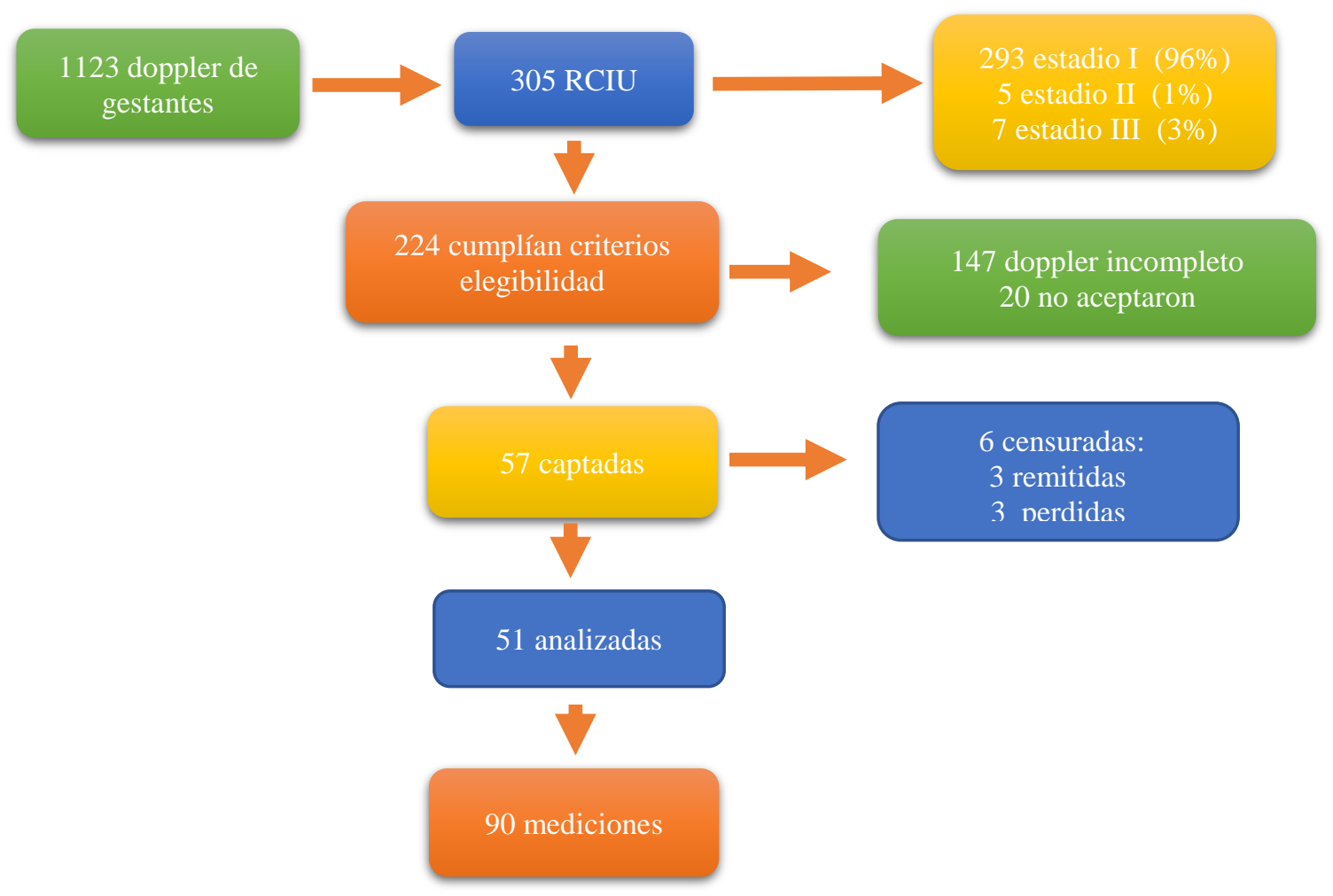

Figura 1: Diagrama de flujo de la población de estudio y la muestra analizada

Tabla 1. Doppler fetal realizados por mes en la unidad maternofetal del Hospital Universitario de Santander en el período de estudio 


\section{JOSHA}

Journal of Science,

Humanities and Arts

\section{DOPPLER FETAL EN EL 2018}

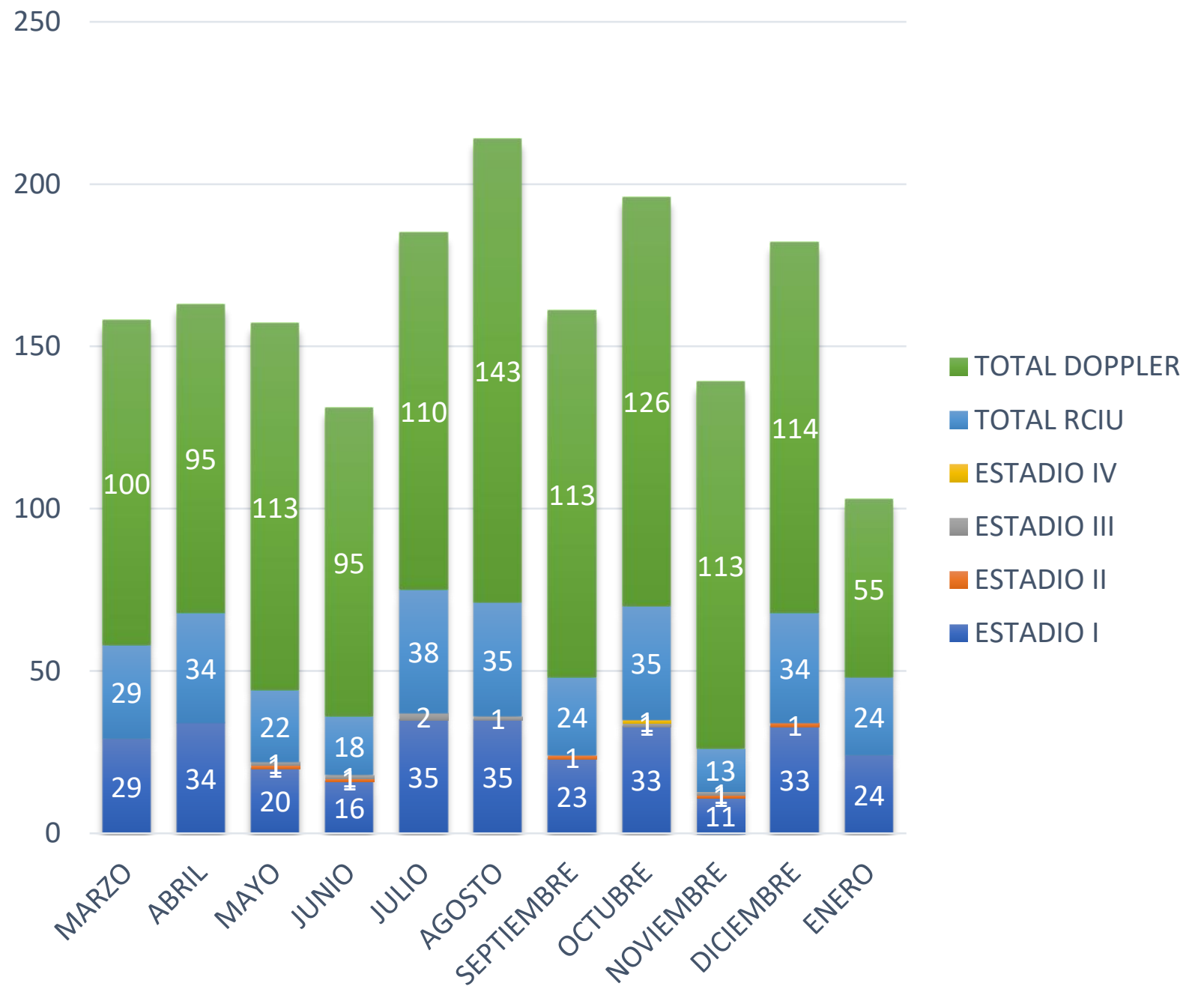

Para el análisis descriptivo se contó con una muestra analítica de 90 mediciones de las 51 pacientes, con una mediana de edad materna de 23 años (RIQ: 18 - 30) y mediana de la edad gestacional de 34,85 semanas (RIQ: 31,5-36,42). La mediana de la paridad fue de 2 gestaciones (RIQ: 1-3); dentro de los desenlaces de las gestaciones previas ninguna relató antecedente de óbito, pero 9 referían antecedente de abortos. 
En tanto a la edad gestacional calculada (fecha de última menstruación confiable o primera ecografía) se notó una correlación del $94.6 \%$ entre la edad estimada por el diámetro transverso del cerebelo medido al ingreso de las gestantes al estudio.

En el análisis descriptivo, en relación al comportamiento de las variables continuas, estas tenían un comportamiento no normal o no paramétrico excepto el IP de la umbilical y el ILA, corroborado por gráficos de cajas y bigotes y pruebas estadísticas como Shapiro-Francia, por lo cual fue necesario trasformar las variables explicatorias, la mejor recomendación fue con el inverso.

Para establecer diferencias en el comportamiento de las diversas variables en los tres grupos de comparación establecidos por estratos según el estadio del RCIU se realizó en el caso de las variables categóricas con la prueba de chi cuadrado de Pearson o la prueba exacta de Fisher, y para las variables no paramétricas se utilizó Kruskal-wallis y Anova para variables continuas normales.

Respecto al incide de TEI; la mediana encontrada fue de 0,51 (RIQ: 0,47-0,54). La mediana del índice de pulsatilidad del istmo aórtico fue de 2,50 (RIQ 2,33-2,72). Por otra parte, la mediana del índice de pulsatilidad del ductus venoso fue de 0,49 (RIQ 0,38-0,64), la mediana del índice de pulsatilidad de la arteria umbilical es 1,09 (RIQ 0,9-1,27), la mediana del índice de pulsatilidad arteria cerebral media es 1,63 (RIQ 1,45-1,92), la mediana de la relación cerebral/umbilical es 1,41 (RIQ 1,12-2,04) y la mediana del índice de pulsatilidad medio de las arterias uterinas fue 0,79 (RIQ 0,69-1,22).

En la siguiente tabla se presentan detalladamente las principales características clínicas.

Tabla 2. Características basales de la población de estudio realizado en la Unidad Materno-fetal del Hospital Universitario de Santander 
Variables

Edad

Número gestaciones

Edad gestacional (EG)

Biometría

Peso estimado al nacer

Percentil del peso

Cerebelo cm

EG cerebelo

EG discreta

Índice de TEI

Percentil TEI

Índice de TEI alterado*

IP Istmo aórtico

Percentil Istmo aórtico

IP uterina derecha

IP uterina izquierda

Media de uterinas

Percentil de uterinas

IP umbilical

Istmo aórtico alterado (SI)*

Uterinas alteradas*

Umbilical alterada*

Flujo reverso umbilical (no)

IP cerebral media

Percentil Cerebral media

Cerebral media alterada*

Relación cerebral umbilical

Relación cerebral umbilical alterada*

Ductus alterado*

FR ductus venoso (no)*

ILA**

ILA alterado*

Estadio doppler*

0

1

2

EG final

Peso RN

Gases de cordón umbilical

realizados*

PH

$\mathrm{BE}^{* *}$ $\mathbf{n}=\mathbf{9 0}$

\begin{tabular}{|c|c|c|}
\hline Mediana & RIQ & $\mathrm{p} ¥$ \\
\hline 23 & $18-30$ & 0,077 \\
\hline 2 & $1-3$ & 0,375 \\
\hline 34,85 & $31,5-36,42$ & 0,395 \\
\hline 31,28 & $28,42-32,57$ & 0,221 \\
\hline 1793,5 & $1290-2050$ & 0,593 \\
\hline 1 & $1-2$ & 0,568 \\
\hline 4,3 & $3,8-4,6$ & 0,412 \\
\hline 34 & $31-36$ & 0,205 \\
\hline 35 & $32-36$ & 0,382 \\
\hline 0,51 & $0,47-0,54$ & 0,142 \\
\hline 70 & $51-87$ & 0,394 \\
\hline 11 & 90 & $<0,01$ \\
\hline 2,5 & $2,33-2,72$ & 0,037 \\
\hline 44 & $24-62$ & 0,111 \\
\hline 0,83 & $0,67-1,06$ & 0,067 \\
\hline 0,78 & $0,65-1,49$ & 0,069 \\
\hline 0,79 & $0,69-1,22$ & 0,086 \\
\hline 78,5 & $52-99$ & 0,823 \\
\hline 1,07 & $0,9-1,27$ & $<0,01$ \\
\hline 4 & 4,44 & $<0,01$ \\
\hline 27 & 30 & $<0,01$ \\
\hline 9 & 10 & $<0,01$ \\
\hline 90 & 100 & $<0,01$ \\
\hline 1,63 & $1,45-1,92$ & $<0,01$ \\
\hline 34,5 & $13-56$ & 0,387 \\
\hline 8 & 8,89 & 0,037 \\
\hline 1,41 & $1,12-2,04$ & $<0,01$ \\
\hline 27 & 30,34 & $<0,01$ \\
\hline 7 & 7,87 & $<0,01$ \\
\hline 90 & 100 & $<0,01$ \\
\hline 11,14 & 3,62 & 0,281 \\
\hline 12 & 13,33 & 0,035 \\
\hline 76 & 84,44 & \\
\hline 6 & 6,67 & \\
\hline 8 & 8,89 & \\
\hline 37 & $35-37$ & $>0,05$ \\
\hline 2072,5 & $1740-2355$ & $>0,05$ \\
\hline 21 & 23,33 & 0,038 \\
\hline 7,29 & $7,26-7,32$ & $>0,05$ \\
\hline$-5,26$ & 2,24 & $>0,05$ \\
\hline
\end{tabular}

Se presentan resultados por columnas.*Frecuencia (porcentaje) **media (desviación estándar) $¥$ Valor de p obtenido mediante la prueba Fisher 
La mediana de edad gestacional a la cual se indicó finalizar la gestación fue de 37 semanas (RIQ 35 - 37); existiendo una diferencia de 2 semanas en la mediana de edad gestacional entre el ingreso al estudio y la terminación del embarazo. Los motivos que llevaron a la finalización de la gestación se enuncian la siguiente tabla:

Tabla 3. Motivos de finalización de la gestación

\begin{tabular}{ccc} 
MOTIVO & NÚMERO & $\mathbf{\%}$ \\
\hline A TERMINO & 46 & 51 \\
\hline PREECLAMPSIA SEVERA & 17 & 20 \\
\hline DOPPLER TIPO III & 12 & 13 \\
\hline OLIGOAMNIOS & 10 & 11 \\
\hline $\begin{array}{c}\text { TRABAJO DE PARTO } \\
\text { PRETERMINO }\end{array}$ & 2 & 2 \\
\hline $\begin{array}{c}\text { RUPTURA PREMATURA DE } \\
\text { MEMBRANAS }\end{array}$ & 1 & 1 \\
\hline ANHIDRAMNIOS & 1 & 1 \\
\hline BRADICARDIA FETAL & 1 & 1 \\
\hline
\end{tabular}

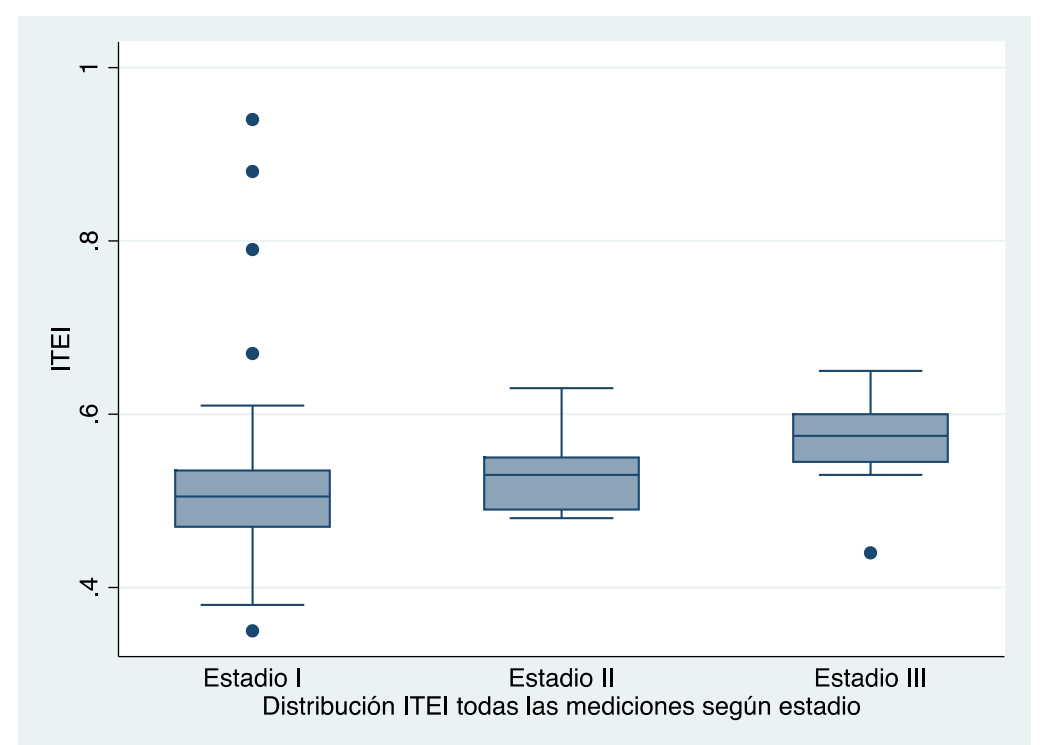

Figura 2: Distribución del Índice de TEI de acuerdo al estadio Doppler 
Se observa en la figura anterior que la mediana del índice de TEI en la población estudiada, va aumentando directamente proporcional al estadio del doppler fetal.

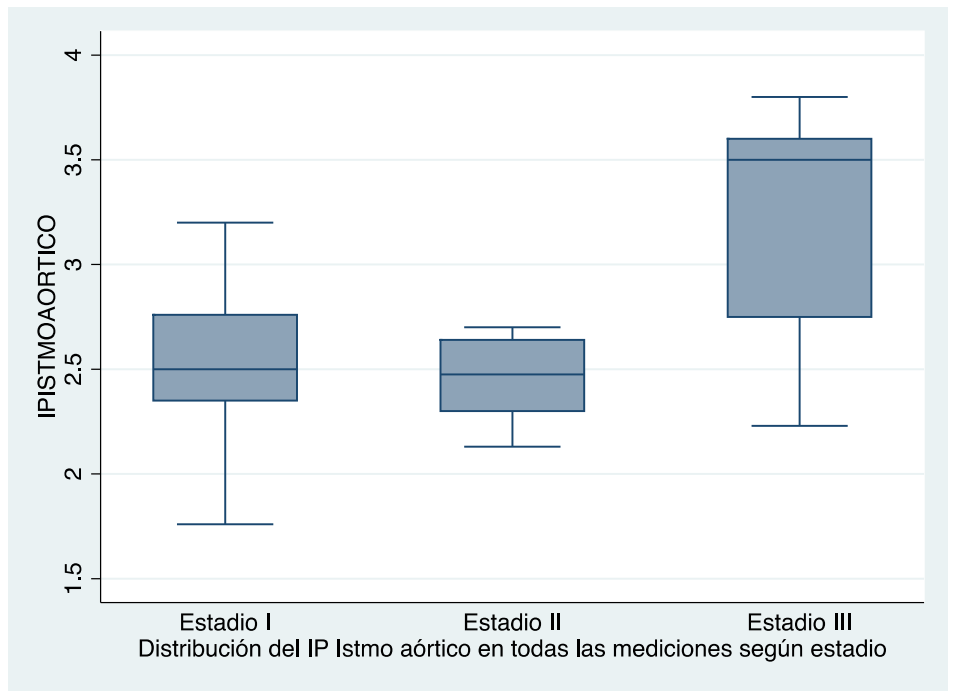

Figura 3: Distribución del istmo aórtico de acuerdo al estadio Doppler

En esta figura se evidencia que en la población estudiada la mediana del IP del istmo aórtico es mayor en el doppler estadio III comparado con la mediana del Doppler estadio I y II.

En la tabla 4 se expone en cada caso estudiado, la evolución de los estadios del doppler a lo largo de la gestación, la evolución de los casos donde inicialmente fueron captadas las pacientes con doppler estadio I con posterior evolución a estadios patológicos (II y III) y se evidencia el momento de nacimiento del feto con RCIU. Además, en la tabla 4 podemos observa los motivos de finalización del embrazo en cada caso del estudio, siendo la principal causa de finalización del embarazo en las pacientes con fetos con RCIU estadio I fue llegar a las 37 semanas (a término) sin repercusiones hemodinámicas, la paciente con estadio II la principal causa fue la preeclampsia severa en 3 pacientes $(50 \%)$, oligoamnios en $2(40 \%)$ y embarazo a termino en la restante (10\%); Las que presentaban fetos con doppler estadio III la alteración del doppler fue el motivo principal de finalización (100\%) (ver tabla 3 y 4). 


\section{JOSHA}

Journal of Science,

Humanities and Arts

Tabla 4. Evolución de los estadios del doppler fetal a lo largo de la gestación, momento y motivo de finalización del embarazo en cada caso estudiado.

\begin{tabular}{|c|c|c|c|c|c|c|c|c|c|c|c|c|c|c|c|}
\hline \multirow[t]{2}{*}{ Pte } & \multicolumn{14}{|c|}{ Semana de gestación } & \multirow[b]{2}{*}{ Motivo de finalización } \\
\hline & 24 & 25 & 26 & 27 & 28 & 29 & 30 & 31 & 32 & 33 & 34 & 35 & 36 & 37 & \\
\hline 1 & & & & & & & & & & & & I & $\mathrm{I}$ & $*$ & A término \\
\hline 3 & & & & & & & & & & & & & & $\mathrm{I} *$ & A término \\
\hline 4 & & & & & & & & & & & & $\mathrm{I} *$ & & & RPM \\
\hline 5 & & & & & & & & & & & $\mathrm{I}$ & $\mathrm{I} *$ & & & Oligoamnios \\
\hline 7 & & & & & & & & & & & & & & $\mathrm{I} *$ & A término \\
\hline 8 & & & & & & & I & & & & & $*$ & & & Oligoamnios \\
\hline 9 & & & & & & & & & & & & II & II & & Oligoamnios \\
\hline 10 & & & & & & & & & & & I & & & $*$ & A término \\
\hline 11 & & & & & & & & & $\mathrm{I} *$ & & & & & & Preeclampsia severa \\
\hline 16 & & & & & & & & & I & & & & I & $\mathrm{I} *$ & Oligoamnios \\
\hline 17 & & & & & & & & & & & & $\mathrm{I}$ & $\mathrm{I}$ & $*$ & A término \\
\hline 18 & & & & & & & & & & & & I & I & $*$ & A término \\
\hline 19 & & & & & & & & & & & & $\mathrm{I}$ & $\mathrm{I}$ & $*$ & A término \\
\hline 20 & & & & & & & & & & & & III & & & Doppler tipo III \\
\hline 21 & & & & & & & & & III & & & & & & Doppler tipo III \\
\hline 22 & & & & & I & & I & III & & & & & & & Doppler tipo III \\
\hline 23 & & & & & & & & & $\mathrm{I} *$ & & & & & & Anhidramnios \\
\hline 24 & & & & & & & & & & & & $\mathrm{I}$ & & $\mathrm{I} *$ & A término \\
\hline 25 & & & & & & & & & & & & & & $\mathrm{I} *$ & Oligoamnios \\
\hline 33 & & & & & & & & & & I & & & & $\mathrm{I} *$ & A término \\
\hline 34 & & & & & & & & & & & & & & $\mathrm{I} *$ & A término \\
\hline 35 & & & & & & & & & & & & & & $\mathrm{I} *$ & A término \\
\hline 36 & & & & & & & & & & & I & & I & $*$ & A término \\
\hline 37 & & & & & & & & & & & & & $\mathrm{I}$ & $\mathrm{I} *$ & A término \\
\hline 38 & & & & & & & & & & & & & $\mathrm{I} *$ & & Oligoamnios \\
\hline 39 & & & & & $\mathrm{I}$ & & & $\mathrm{I}$ & $\mathrm{I} *$ & & & & & & Preeclampsia severa \\
\hline 40 & & & & & & & & & I & & & & & $\mathrm{I} *$ & A término \\
\hline 41 & & & & & & & & & & & & & & $I *$ & A término \\
\hline 42 & & & & & & & & & & & I & & I & $\mathrm{I} *$ & A término \\
\hline 43 & & & & & & & & & & & & $\mathrm{I}$ & $\mathrm{I}$ & $\mathrm{I} *$ & A término \\
\hline 44 & & & & & & & & & & & I & $\mathrm{I}$ & I & $\mathrm{I} *$ & A término \\
\hline 45 & & & & & & & & & & & & $\mathrm{I} *$ & & & Bradicardia fetal \\
\hline 46 & & & & & & & & & & & & & I & $\mathrm{I} *$ & A término \\
\hline 47 & & & & & & & & & & & & & & $\mathrm{I} *$ & A término \\
\hline 48 & & & III & & & & III & & & & & & & & Doppler tipo III \\
\hline 49 & & & & & & & I & $\mathrm{I} *$ & & & & & & & TPP \\
\hline 50 & & & & & & & & & & & & & & $\mathrm{I} *$ & A término \\
\hline 51 & & & & & & I & I & & & & & III & & & Doppler tipo III \\
\hline
\end{tabular}

Se presentan resultados por columnas. * Momento del nacimiento 
\begin{tabular}{ll} 
Estadio I & I \\
\cline { 2 - 2 } Estadio II & II \\
Estadio III & III
\end{tabular}

Los parámetros para evaluar asfixia perinatal fueron la alteración de gases arteriales, lo cual no se evidencio en ningún paciente, teniendo en cuenta que la medición solo se le realizó en 21 pacientes que corresponde al 23,33\% de las mediciones, de los cuales 15 son estadio I, 4 estadio II y 2 estadio III; la mediana el PH de sangre del cordón umbilical fue de 7.29 RI (7.26 - 7.21), base exceso mediana -4.9 (RIQ -7.1 - -3.1), sin embargo a las pacientes con doppler fetal alterados en estadio II y III se les evaluó adicionalmente al recién nacido el APGAR para evaluar asfixia y todos presentaron resultados dentro de los limites normales. Destacando, además, que en ningún doppler evaluado se evidenció flujo reverso en las diferentes variables y el desenlace fetal fue satisfactorio ya que todos nacieron vivos y sin parámetros de asfixia.

Por otro lado, en el análisis comparativo entre la presencia de alteración de el istmo aórtico y los estratos del doppler, se óque el estadio III presentaba mayor tendencia de tener alteraciones del istmo. Igual tendencia se encontró con las arterias uterinas alteradas, la arteria umbilical alterada, la arteria cerebral media alterada, el ductus alterado, el ILA alterado y los gases alterados. No se encontró una relación estadísticamente significativa con la edad de la gestante.

\section{CASOS CON DOPPLER ESTADIO II Y III}

Se realizó un análisis de subgrupos con una muestra analítica de 24 mediciones de las gestantes que llegaron en el seguimiento a estadios II y III. La mediana de edad de las participantes del estadio I fue de 22,5 años (RIQ 19-34,5), de 19.5 años (RIQ 18-23) en el estadio II y de 24 años (RIQ 22-23) en el estadio III, a su vez, la mediana de la edad gestacional de 32 semanas (RIQ: 30,35-35,07) en el estadio I, de 35.85 semanas (RIQ: 34.7136.57) en el estadio II y de 31.57 (RIQ: 30,71-34.54) semanas en el estadio III. La mediana 


\section{JOSHA}

Journal of Science,

Humanities and Arts

del peso estimado al nacer de los recién nacidos fue de 1260 (RIQ: 1010,5-1717,5) del estadio I, de 1704 (RIQ: 1227-1720) del estadio II y de 1032 (RIQ: 749-1748) del estadio III. Es importante mencionar que no se presentaron casos relacionados con abortos, óbitos ni nacidos muertos (ver tabla 5).

Tabla 5. Análisis exploratorio con características clínicas de los participantes discriminado de acuerdo con los tres estadios Doppler estudiados (I, II y III).

\begin{tabular}{|c|c|c|c|c|c|c|c|c|c|}
\hline \multirow{3}{*}{ Variables } & \multicolumn{3}{|c|}{ Estadio I } & \multicolumn{3}{|c|}{ Estadio II } & \multicolumn{3}{|c|}{ Estadio III } \\
\hline & & $n=10$ & & & $n=6$ & & & $n=8$ & \\
\hline & Mediana & RIQ & $\mathrm{p} ¥$ & Mediana & RIQ & $\mathrm{p} ¥$ & Mediana & RIQ & $\mathrm{p} ¥$ \\
\hline Edad & 22,5 & $19-34,5$ & & 19,5 & $18-23$ & & 24 & $22-23$ & \\
\hline $\begin{array}{l}\text { Edad } \\
\text { gestacional } \\
(\mathbf{E G})\end{array}$ & 32 & $\begin{array}{c}30,35- \\
35,07\end{array}$ & $>0,05$ & 35.85 & $\begin{array}{l}34.71- \\
36-57\end{array}$ & $>0.05$ & 31.57 & $\begin{array}{c}30.71- \\
34.54\end{array}$ & $>0.05$ \\
\hline Biometría & 28,28 & $\begin{array}{c}27,07- \\
30,85\end{array}$ & $>0,05$ & 30.78 & $\begin{array}{l}28.14- \\
32.14\end{array}$ & $>0.05$ & 27.74 & $\begin{array}{l}25.75- \\
31.28\end{array}$ & $>0.05$ \\
\hline $\begin{array}{l}\text { Peso estimado al } \\
\text { nacer }\end{array}$ & 1260 & $\begin{array}{l}1010,5- \\
1717,5 \\
\end{array}$ & $>0,05$ & 1704 & $\begin{array}{l}1227- \\
1720\end{array}$ & $>0.05$ & 1032 & $\begin{array}{l}749- \\
1748 \\
\end{array}$ & $>0.05$ \\
\hline Cerebelo cm & 3,93 & $3,78-4,3$ & $>0,05$ & 4.45 & $4.27-4.7$ & $>0.05$ & 3.9 & $3.8-4.2$ & $>0.05$ \\
\hline EG Cerebelo & 31,5 & $30-34$ & $>0,05$ & 35 & $34-37$ & $>0.05$ & 31 & $30.5-34$ & $>0.05$ \\
\hline EG discreta & 32 & $30-35$ & $>0,05$ & 35.5 & $35-37$ & $>0.05$ & 31.5 & $30.5-37$ & $>0.05$ \\
\hline EG final & 35,42 & $34,71-40$ & $>0,05$ & 36.14 & $35.42-37$ & $>0,05$ & 31.64 & $\begin{array}{l}30.71- \\
34.75 \\
\end{array}$ & $>0,05$ \\
\hline Peso RN & 1845 & $1740-2870$ & $>0,05$ & 1937 & $\begin{array}{l}1670- \\
2030\end{array}$ & $>0,05$ & 962 & $\begin{array}{l}860- \\
1720\end{array}$ & $>0,05$ \\
\hline $\mathbf{P H}$ & 7,23 & $7,15-7,31$ & $>0,05$ & 7.24 & $7.2-7.28$ & $>0,05$ & 1.22 & $\begin{array}{l}1.15- \\
1.29\end{array}$ & $>0,05$ \\
\hline $\mathrm{BE}$ & $-7,05$ & $\begin{array}{c}-9,02-- \\
4,09\end{array}$ & $>0,05$ & -5.1 & $-5.1--4.9$ & $>0,05$ & -5.7 & $\begin{array}{l}-9.2-- \\
2.2\end{array}$ & $>0,05$ \\
\hline
\end{tabular}

Se presentan resultados por columnas.

$¥$ Valor de p obtenido mediante la prueba Kruskal-Wallis.

En las pruebas estadísticas aplicadas en el análisis exploratorio se encontraron relaciones estadísticamente significativas entre las siguientes variables y el estadio Doppler: índice de TEI, IP del istmo aórtico, IP de la arteria umbilical, IP de la arteria cerebral media, la relación cerebral umbilical, el IP del ductus alterado e ILA. A continuación, se presentan detalladamente las principales características clínicas de los participantes discriminado de acuerdo a cada uno de los estadios Doppler (ver tabla 6) 


\section{JoSHA}

Journal of Science,

Humanities and Arts

Tabla 6. Análisis exploratorio con variables del doppler fetal discriminado de acuerdo con los tres estadios Doppler estudiados (I, II y III)

\begin{tabular}{|c|c|c|c|c|c|c|c|c|c|}
\hline \multirow{3}{*}{ Variables } & \multicolumn{3}{|c|}{ Estadio I } & \multicolumn{3}{|c|}{ Estadio II } & \multicolumn{3}{|c|}{ Estadio III } \\
\hline & \multicolumn{3}{|c|}{$\mathbf{n}=\mathbf{1 0}$} & \multicolumn{3}{|c|}{$n=6$} & \multicolumn{3}{|c|}{$n=8$} \\
\hline & Mediana & RIQ & $\mathrm{p} ¥$ & Mediana & RIQ & $\mathrm{p} ¥$ & Mediana & RIQ & $\mathrm{p} ¥$ \\
\hline Índice de TEI* & 0,51 & 0,074 & $<0.05$ & 0.53 & $\begin{array}{l}0.49- \\
0.55 \\
\end{array}$ & $<0.05$ & 0.57 & $\begin{array}{c}0.54- \\
0.6 \\
\end{array}$ & $<0.05$ \\
\hline $\mathrm{P} \mathrm{TEI}^{+}$ & 67,79 & 26,51 & $<0,05$ & 65.5 & $47-72$ & $<0.05$ & 97 & $96-98$ & $<0.05$ \\
\hline Istmo aórtico $^{+}$ & 2,76 & 0,52 & $<0,05$ & 2.4 & $2.3-2.6$ & $<0.05$ & 3.5 & $2.7-3.6$ & $<0.05$ \\
\hline P Istmo aórtico * & 56,37 & 29,76 & $>0,05$ & 45.5 & $22-63$ & $>0.05$ & 97 & $57-98$ & $>0.05$ \\
\hline P Uterina & 99 & 99-99 & $>0,05$ & 89.5 & 74-99 & $>0.05$ & 99 & $96-99$ & $>0.05$ \\
\hline IP umbilical+ & 1,082 & 0,32 & $<0,05$ & 1.55 & $\begin{array}{l}1.25- \\
1.65 \\
\end{array}$ & $<0.05$ & 1.55 & $\begin{array}{l}1.32- \\
1.61 \\
\end{array}$ & $<0.05$ \\
\hline P umbilical & 45,5 & $44-65$ & $>0,05$ & 95 & $83-96$ & $>0.05$ & 93 & $87-97$ & $>0.05$ \\
\hline IP cerebral media ${ }^{+}$ & 1,73 & 0,21 & 0,03 & 1.47 & $\begin{array}{l}1.28- \\
1.57 \\
\end{array}$ & 0,03 & 1.39 & $\begin{array}{l}1.27- \\
1.56 \\
\end{array}$ & 0,03 \\
\hline$P$ cerebral & 39 & $14-51$ & $>0,05$ & 10 & $6-75$ & $>0,05$ & 5 & $3.5-14$ & $>0,05$ \\
\hline $\begin{array}{l}\text { Relación cerebral } \\
\text { umbilical }\end{array}$ & 1,66 & $\begin{array}{l}1,3- \\
2,04 \\
\end{array}$ & $<0,05$ & 1.15 & $\begin{array}{c}0.95- \\
1.4 \\
\end{array}$ & $<0,05$ & 0.96 & $\begin{array}{c}0.82- \\
1.09 \\
\end{array}$ & $<0,05$ \\
\hline $\begin{array}{l}\text { P relación cerebral } \\
\text { umbilical }\end{array}$ & 33 & $7-55$ & $>0,05$ & 8 & $1-55$ & $>0,05$ & 1 & $1-2$ & $>0,05$ \\
\hline IP ductus venoso & 0,47 & 0,26 & $>0,05$ & 0.51 & $\begin{array}{c}0.63- \\
0.34\end{array}$ & $>0,05$ & 0.89 & $\begin{array}{c}0.79- \\
1.05\end{array}$ & $>0,05$ \\
\hline P ductus venoso & 57 & $8-68$ & $<0,05$ & 48 & $12-78$ & $<0,05$ & 98 & $96-99$ & $<0,05$ \\
\hline ILA & 12,03 & $11-14$ & $<0,05$ & 3.7 & $3-6$ & $<0,05$ & 11.45 & $8.4-12$ & $<0,05$ \\
\hline
\end{tabular}

Se presentan resultados por columnas.

*Media (Desviación estándar) ¥ Valor de p obtenido mediante la prueba Kruskal-Wallis.

+valor p obtenido por la prueba chi2 de Pearson

\section{Análisis de correlación de Spearman y de Pearson}

Teniendo en cuenta todas las pacientes que tenían al menos una medición (51 pacientes), la correlación de Spearman entre el índice de TEI y el Istmo aórtico es del 17,07\% ,es decir débil pero positiva y no significativa (p 0,23), sin embargo, estratificado por estadios de RCIU por doppler esta relación aumenta; siendo en el estadio I la correlación de 4,94\% (p 0,75), en el estadio II es de $1 \%(\mathrm{p}<0.001)$ y en el estadio III $80 \%$ (p 0,20). Ahora bien, dado que el comportamiento descrito previamente del TEI y del Istmo aórtico era de no normalidad, se establece la correlación con las variables normalizadas ,obteniendo una correlación del inverso del índice de TEI y del inverso del Istmo aórtico en el estadio I 


\section{JOSHA}

Journal of Science,

Humanities and Arts

positiva en el 3,29\% en el estadio II positiva de $38,08 \%$ y el en estadio III aumenta dicha correlación a 73,86\%.

Se pudo evidenciar en la población analizada, que existe una correlación lineal positiva entre el inverso del índice de pulsatilidad del istmo aórtico fetal y el inverso de la función del ventrículo izquierdo. Es decir, a medida que aumenta el istmo aórtico aumenta el ITEI.

$\mathrm{Al}$ analizar cada uno de los estadios se pudo estimar un coeficiente de determinación (R2) de: 0.001 para el estadio I, de 0.13 para el estadio II y de 0.54 para el estadio III. De acuerdo con el valor de coeficiente de determinación $\left(\mathrm{R}^{2}\right)$ la proporción de varianza es compartida por ambas variables, siendo para el estadio I pequeña, en el estadio II media/moderada y en el estadio III fuerte (ver figura 4).

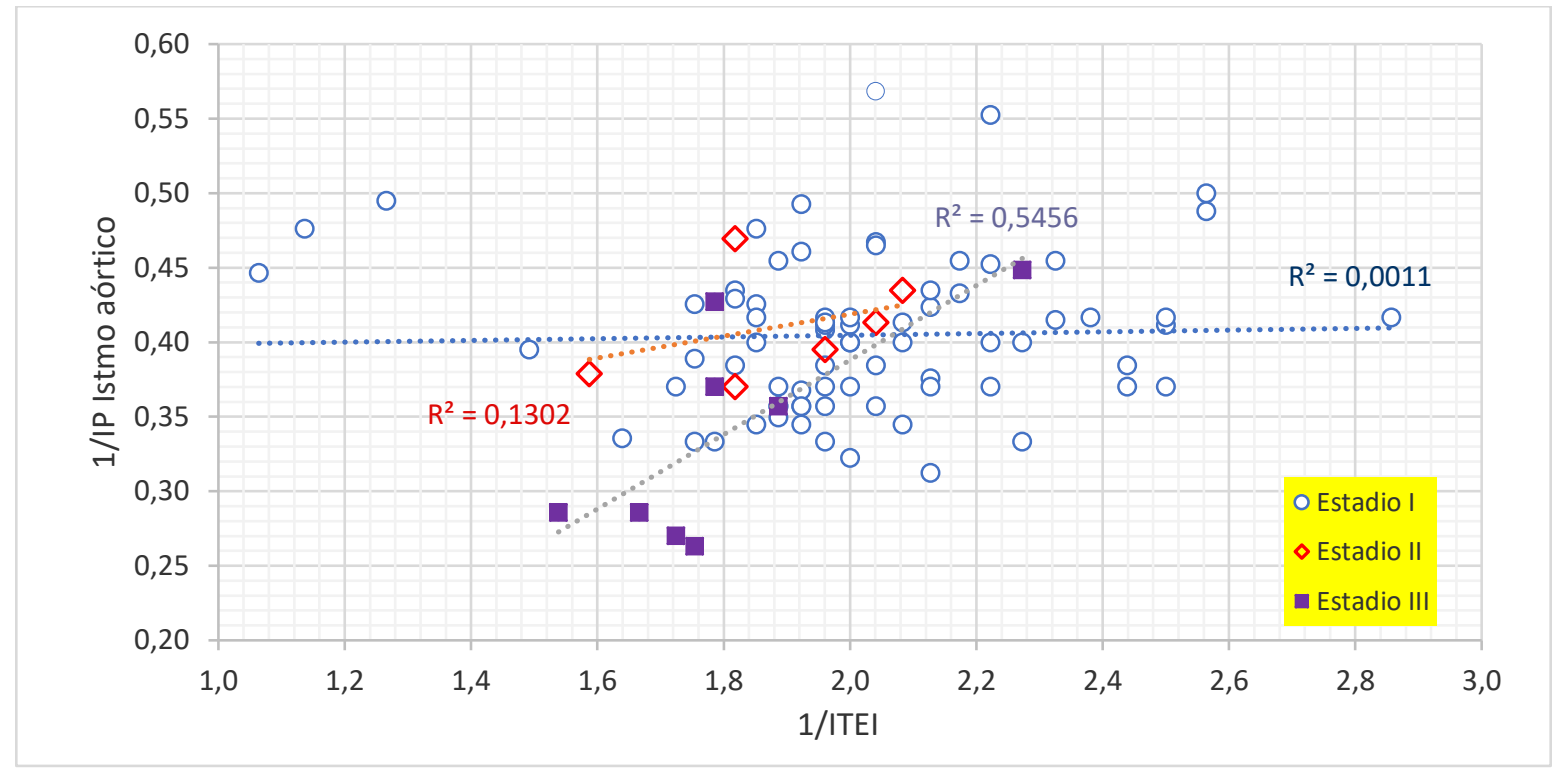

Figura 4: Gráfica de correlación entre el istmo aórtico y el ITEI

\section{DISCUSIÓN}

Nuestro estudio nos permite observar que existe una relación lineal entre el inverso del IP del istmo aórtico y el inverso de la función del ventrículo izquierdo medido con el índice de 
TEI, esta correlación incrementa cuando se estratifica según el estadio de RCIU definido por las medidas del doppler, siendo fuerte en los estadios III; estos resultados son acordes con los evaluados por R. Cruz - Martínez et al.(32) en su estudio donde también se evidenció aumento de la correlación a medida que aumenta el estadio patológico del doppler fetal. Además, mostraron una proporción significativamente mayor de aumento de ITEI (28.1 vs. 6.7\%; P <0.01) y anormal de IP del istmo aórtico (14.6 vs. $5.1 \%$; P <0.01) lo que sigue reafirmando la relación que existe.

Existe una relación lineal entre el índice de TEI y el istmo aórtico analizados en el estadio Doppler II y III respectivamente, lo que nos hace sugerir que pudiese ser predictivo para los fetos con RCIU y doppler anormal, siendo esto similar a lo evidenciado en el estudio de Del Río M et al.(33) donde de 51 fetos con RCIU severo detectó el resultado perinatal adverso predicho adecuadamente por un aumento del IP del istmo aórtico (área bajo la curva 0,77; IC del 95\%, 0,63-0,92; P <0,005).

Una de las principales fortalezas del estudio es que es pionero en establecer correlación entre los valores del índice de pulsatilidad del istmo aórtico fetal y del indice de TEI en fetos con RCIU severo dado por peso fetal estimado por debajo del percentil 3 (PFE $<$ p3), ya que generalmente se estudiaba población con percertil menor de 10 con doppler alterado o fetos pequeños para la edad gestacional.

Los estudios (8)(33) han encontrado una correlación significativa ( P <0,001) entre el flujo sanguíneo retrógrado en el istmo aórtico y el resultado perinatal adverso, siendo la mortalidad perinatal general más alta en el grupo del flujo retrógrado ( $70 \%$ vs. 4,8\%, P <0,001), destacando que en la muestra estudiada en nuestro trabajo no fue posible analizar el resultado fetal adverso ya que en ningún doppler evaluado se evidenció flujo retrógrado en las diferentes variables y el desenlace fetal fue satisfactorio ya que todos nacieron vivos y con parámetros de asfixia negativos. Entre las limitaciones del estudio, además de ser solo descriptivo, tener un tamaño de muestra insuficiente y no disponer de otras variables importantes como la toma en forma rutinaria de gases arteriales a todos los cordones umbilicales de los recién nacidos, porque no está protocolizado por el servicio de ginecología 
dentro del Hospital Universitario de Santander; por eso este trabajo propone como recomendación la toma rutinaria de gases arteriales por parte del ginecólogo en el momento de pinzamiento del cordón, a todos los recién nacidos con RCIU para descartar asfixia perinatal. De igual manera, se desconoce presencia de sesgos de medición ya que no fue posible establecer la concordancia entre evaluadores, debido a que varios de los miembros de la unidad se encuentran en una curva de aprendizaje.

Por último, resultó evidente que era necesario un tamaño de muestra suficiente para lograr las estimaciones pretendidas, impidiendo aplicar un verdadero modelo predictivo, ya que la prevalencia de RCIU de nuestra institución con estadios de Doppler patológicos II, III y IV es baja. Sin embargo, se pudieron encontrar tendencias de relación entre las variables medidas por medio de pruebas estadísticas exploratorias. Por lo tanto, se destaca de nuevo, que se deben realizar estudios colaborativos interinstitucionales, aumentar el tamaño de muestra y el tiempo de observación, ya que de esta manera se podrían evaluar las asociaciones previstas.

\section{CONCLUSIÓN Y RECOMENDACIONES}

Esta investigación pudo evidenciar que existe una correlación positiva entre los valores del índice de pulsatilidad del istmo aórtico fetal y de la función del ventrículo izquierdo en fetos con peso fetal estimado por debajo del percentil 3 (PFE < p3). A su vez, se encontró que a medida que incrementa el estadio Doppler hay un aumento en la correlación entre las dos variables analizadas.

Es importante destacar que este estudio permitió establecer la prevalencia de RCIU de $27,15 \%$ en nuestra institución, lo que aviva el desarrollo de posteriores análisis para continuar con la investigación de los criterios que puedan establecer cuando el feto se encuentra sobre el límite de la descompensación cardiaca. En este sentido, el análisis comparativo en los valores de la onda de flujo del istmo aórtico, junto con los cambios iniciales en la función cardiaca debe continuarse como línea de investigación con esta condición Materno-Fetal, con 
trabajos donde se cuente con un apropiado tamaño de muestra considerando la baja prevalencia de la enfermedad en nuestra población; incluyendo también, todas las variables reconocidas y determinar una relación causal controlando la confusión. El hecho de confirmar estas asociaciones a nivel clínico nos permite generar protocolos de manejo para nuestra población.

Adicionalmente teniendo en cuenta nuestros resultados, se recomienda considerar como protocolo de seguimiento a las pacientes con RCIU severo el IP del istmo aórtico, el índice de TEI y la toma de gases arteriales al nacer para optimizar el diagnóstico temprano e identificación de compromiso fetal precoz y realizar un curso de formación en doppler para la realización de estos parámetros en nuestra institución para que dichas medidas sean realizadas por todo el personal de la unidad maternofetal del Hospital Universitario de Santander.

\section{BIBLIOGRAFÍA}

1. Callen P. Ecografia En Obstetricia Y Ginecologia [Internet]. Fifth Edit. Saunders, editor. Philadelphia: Elsevier Inc; 2009. 1010 p. Available from: https://ezproxy.uis.edu.co:2864/\#!/browse/book/3-s2.0-C20090423298

2. Cruz-Martinez R, Figueras F, Hernandez-Andrade E, Oros D, Gratacos E. Changes in myocardial performance index and aortic isthmus and ductus venosus Doppler in term, small-for-gestational age fetuses with normal umbilical artery pulsatility index. Ultrasound Obstet Gynecol. 2011;38(4):400-5.

3. Acharya G. Technical aspects of aortic isthmus Doppler velocimetry in human fetuses. Ultrasound Obstet Gynecol. 2009;33(6):628-33.

4. Fouron JC. The unrecognized physiological and clinical significance of the fetal aortic isthmus. Ultrasound Obstet Gynecol. 2003;22(5):441-7.

5. Figueras F, Benavides A, Del Rio M, Crispi F, Eixarch E, Martinez JM, et al. 


\section{JOSHA}

Journal of Science,

Humanities and Arts

Monitoring of fetuses with intrauterine growth restriction: Longitudinal changes in ductus venosus and aortic isthmus flow. Ultrasound Obstet Gynecol. 2009;33(1):39_ 43.

6. Kennelly MM, Farah N, Hogan J, Reilly A, Turner MJ, Stuart B. Longitudinal study of aortic isthmus Doppler in appropriately grown and small-for-gestational-age fetuses with normal and abnormal umbilical artery Doppler. Ultrasound Obstet Gynecol. 2012;39(4):414-20.

7. Del Río M, Martínez JM, Figueras F, Bennasar M, Olivella A, Palacio M, et al. Doppler assessment of the aortic isthmus and perinatal outcome in preterm fetuses with severe intrauterine growth restriction. Ultrasound Obstet Gynecol. 2008;31(1):41-7.

8. Cruz-Martinez R, Figueras F, Benavides-Serralde A, Crispi F, Hernandez-Andrade E, Gratacos E. Sequence of changes in myocardial performance index in relation to aortic isthmus and ductus venosus Doppler in fetuses with early-onset intrauterine growth restriction. Ultrasound Obstet Gynecol [Internet]. 2011 Aug 1 [cited 2019 Jan 22];38(2):179-84. Available from: http://doi.wiley.com/10.1002/uog.8903

9. Del Río M, Martínez JM, Figueras F, Bennasar M, Olivella A, Palacio M, et al. Doppler assessment of the aortic isthmus and perinatal outcome in preterm fetuses with severe intrauterine growth restriction. Ultrasound Obstet Gynecol [Internet]. 2008 Jan 1 [cited 2019 Jan 22];31(1):41-7. Available from: http://doi.wiley.com/10.1002/uog.5237 


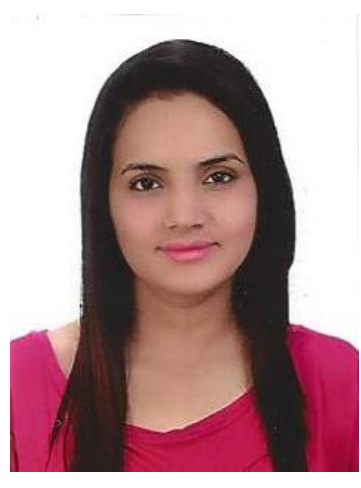

MALKA IRINA WADNIPAR GUTIÉRREZ es especialista en Ginecología y Obstetricia de la Universidad Industrial de Santander, Bucaramanga, Colombia. Estudió medicina en la Universidad del Magdalena, Santa Marta, Colombia, con promedio académico de estudiante distinguido. Autora principal del estudio: "Asociación entre el índice de pulsatilidad del istmo aórtico y la función cardíaca del ventrículo izquierdo en fetos por debajo del percentil tres para la edad gestacional", artículo de investigación realizado que sirvió como tesis para la obtención de su título de especialista. / Is a specialist in Gynecology and Obstetrics at the Universidad Industrial de Santander, Bucaramanga, Colombia. She studied medicine at the Universidad de Magdalena, Santa marta, Colombia. Main author of the study:" Association between the pulsatility index of the aortic isthmus and the cardiac function of the left ventricle in fetos below the percentile three for the gestational age.", article of research carried out that served as thesis for obtaining its specialist title. 